\title{
User experience in an interactive music virtual reality system: an exploratory study
}

\author{
Thomas Deacon, Tony Stockman, and Mathieu Barthet* \\ Centre for Digital Music, Queen Mary University of London \\ t.e.deacon@qmul.ac.uk
}

\begin{abstract}
The Objects $V R$ interface and study explores interactive music and virtual reality, focusing on user experience, understanding of musical functionality, and interaction issues. Our system offers spatiotemporal music interaction using 3D geometric shapes and their designed relationships. Control is provided by tracking of the hands, and the experience is rendered across a head-mounted display with binaural sound presented over headphones. The evaluation of the system uses a mixed methods approach based on semi-structured interviews, surveys and video-based interaction analysis. On average the system was positively received in terms of interview self-report, metrics for spatial presence and creative support. Interaction analysis and interview thematic analysis also revealed instances of frustration with interaction and levels of confusion with system functionality. Our results allow reflection on design criteria and discussion of implications for facilitating music engagement in virtual reality. Finally our work discusses the effectiveness of measures with respect to future evaluation of novel interactive music systems in virtual reality.
\end{abstract}

Keywords: creativity support, design research, flow, interaction analysis, interactive music systems, thematic analysis, user experience, virtual reality

\section{Introduction}

Virtual Reality (VR) is now an accessible medium for music interaction, that could transform consumption and production of music, if designed systems can capitalise on the unique possibilities of VR. For those without musical experience it could provide new forms of creative engagement while for those more experienced the medium offers new possibilities for creating and interacting with music. New media formats have continually, and drastically augmented the music creation and listening landscape 10. As a new medium VR needs adoption, requiring effective design of the technology to foster a constructive dialogue

\footnotetext{
* This is a post-peer-review, pre-copyedit version of an article published in the Lecture Notes in Computer Science (LNCS) volume 10525 ("Bridging People and Sound"), pp.192-216, Springer, 2017. The final authenticated version is available online at: https://doi.org/10.1007/978-3-319-67738-5_12
} 
around music interaction as a non-specialist pastime. Changing the relationship between producer, consumer, and creator alters our relationship to music and could build towards increased engagement in music with those without extensive musical experience [4].

As a medium VR is visual, sonic and inherently interactive. Designing an interactive music system for this medium requires new understanding given the potentially unique characteristics of musical interactions occurring within VR, "warranting their own performance practices, approaches and taxonomies" [17. Of the research conducted into direct control of interactive music systems in VR categories include: (i) virtual object manipulation with parametrised sound output [24], (ii) controlling musical characteristics of a pre-existing composition [26], (iii) virtual instrument representations [15, (iv) spatial audio interfaces [38, (v) multi-process audio environments 6] that are combinations of the previous categories (this includes Objects VR). Many of these implementations offer novel interaction methods coupled with creative feedback and visualisation but give little analysis of user skill levels, and how this alters individual experience and results.

Our Objects $V R$ interface design explores music interaction in an interactive music system in virtual reality (VR). In Objects $V R$ the user interacts with an object-based interface to control three tracks of music (drums, bass and synth) and track-based effects, see section 3.2. Hand actions are captured through a Leap Motion device attached to an Oculus Rift DK2 head-mounted display (HMD) that renders the VR environment. We investigate users' natural exploration and formation of understanding to be able to interact creatively in the proposed virtual environment. We use an exploratory mixed methods assessment of user exploration behaviours, subjective experience and understanding. The study compares users' expertise with music technology and sound synthesis using thematic analysis [9], interaction analysis [23, a creativity support metric [11], a spatial presence survey [18] and a sonic interaction survey. Discussion puts forward relevant research directions to support creative engagement with interactive music systems in VR by understanding different groups experience; this informs design by understanding how users naturally engage and understand a VR interactive music system. Though this design research can only address the proposed music interfaces particular functionality and chosen musical genre, further developments could inform design principles for other novel interactive music applications. Effective evaluation facilitates design development of prototypes and artefacts, and more generally, research into key theories around music interaction.

\section{Related Works}

A common factor in measuring the effectiveness of VR is the concept of presence. An operational definition of Spatial Presence is that it is a binary experience, where perceived self-location, action possibilities and mental capacities are connected to a mediated spatial environment instead of reality [37]. Further analysis 
of the Spatial Presence theory highlights that if affordances of virtual objects activate actions then a feeling of presence is fed back [29]. This means that within an experience it is not only the spatial environment that a user feels present but also the virtual object framework.

Encouraging creative music engagement is a suitable goal for any interactive music system. The theory of flow describes states of optimal experience that can improve engagement $[12$. Attaining a state where users can perform supremely and effortlessly involves balancing of motivation and skill; based on the design of: challenge, learning, and functionality 27]. So, for new interactive music systems in VR, at any skill level, flow should be actively positioned in design and evaluation phases. Flow states have the following conditions: (i) clear goals, (ii) unambiguous feedback on performance, (iii) balance of challenge and ability; and effects: (i) action and awareness merge, (ii) total concentration, (iii) sense of control, (iv) self-consciousness disappears, (v) distorted sense of time, (vi) autotelic experience.

The exploratory nature of this work means qualitative methods are suitable for developing understanding within the design space, such as discourse analysis of live music-making using interactive systems [34]; research of new musical instrument design using grounded theory [22]; and use of thematic analysis to analyse design decisions effects on sonic exploration and manipulation [1]. We deem it important not to focus purely on usability, as this does not account for the diverse nature of experience with musical interfaces. In the design of user experience studies, using professional musicians, Johnston suggests three important questions 22]: Do instruments meet design criteria identified during design? How do musicians experience them? What is the relationship between the instrument and the musician's experience?

In this study, the performance of the rendered environment is evaluated using spatial presence conceptualisations. Flow theory is used a heuristic to analyse users' situated interactions and conceptual understanding using interaction analysis, interview thematic analysis, and survey self-report.

\section{Design Space}

The formulation of problems and solutions in the creation of the system is the design space, and inside this space all the competing theories and influences merge. The design space is a conceptual tool to accommodate multiple disciplines and approaches in representing the design work. But the design space cannot be fully described here because of its complexity and size. We used a user-centred research through design methodology 39 for the production of prototypes and artefact 1 . The design process is based on research and practise of prototyping [20], testbed comparisons [8], heuristic evaluation [35] and user experience testing [14]. Similar approaches have been used to design music technology for a gesturally controlled operatic-lead signal processor 13 and a digitally augmented cello 2 .

\footnotetext{
${ }^{1}$ We define prototype as any representation of a design idea, regardless of medium, and artefact as the interactive system being designed 20 .
} 


\subsection{Design Criteria}

The design criteria were that the VR experience should: (i) Be accessible and fun for novices of music technology, musical education, and VR; (ii) Allow 'physical' experimentation and interaction with musical content; (iii) Lead to high levels of presence; (iv) Allow users to experience flow states. The focus on novice engagement means levels of musical control must be carefully considered. It looks to provide novice users the ability to learn purely within the experience, and maintain coherent musical output e.g. stable harmony and well fitted rhythm. Body-based interaction can provide an immersive presence enhancing interaction in VR [32, while engaging users in embodied musical interaction [16]. Also engaging with fluid arm motions has been shown to improve creativity [33, making body-based spatial interaction a field of worthwhile development for musical engagement. By positioning flow in the design space we are hoping to create a quality of interaction that promotes learning for novices but can also provides experts with new ways of interacting or feeling creative. For novices we want "good flow" 28, experiences that can scaffold towards deeper musical engagement and experience, not just an immediate feelings of pleasing perceptual stimulus and enjoyment.

\subsection{Artefact}

Designing the relationship between geometry, motion and sound is essential to Objects $V R$ interface. Understanding of sonic functionality is gained while exploring a VR environment, where the environment is the interface. The system allows control of playback of short repetitive musical phrases of a 2-step UK garage and dubstep style. This is combined with spatial audio panning, and timbral control utilising gestural interaction and direct interface manipulation. A composition was commissioned to be easily layered in many different ways ${ }^{2}$

The system utilises grabbing and contact interactions in an object-based interface to control three tracks of music (drums, bass and synth) and track-based effects. Users' actions are captured through a Leap Motion device attached to an Oculus Rift DK2 head-mounted display (HMD) that renders the VR environment. The VR application sends OSC data about user action, objects, and system states to Ableton Live and Max/MSP. Audio loops are triggered directly through the LiveAPI using javascript commands, while object parameters are remotely controlled through various mappings, discussed in the next paragraph. Ambisonic to binaural panning is rendered using the Higher Order Ambisonics library for Max/MSP 30].

Interface objects The visual design of object form represents context of music interaction: effects control or loop playback. Colour represents content relationships to track type, indicated in figure 1. A Grid Unit Menu (GUM) is a container object with a series of buttons that allow triggering of loop content.

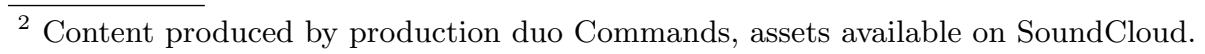




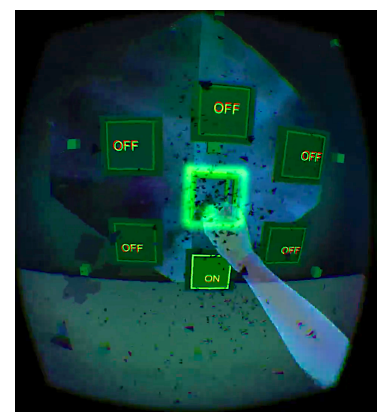

(a) Green Synth Grid Unit Menu with Prism Explosion Feedback

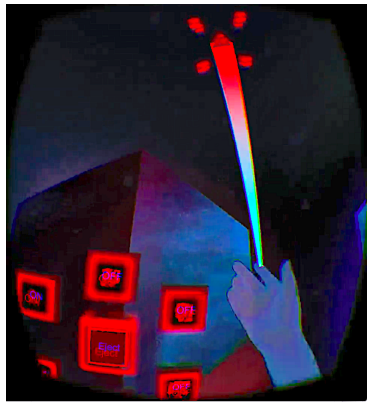

(b) Red Drum Prism Grab Attempt with Ray casting Beam

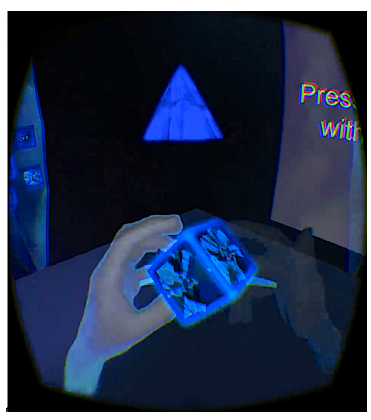

(c) Blue Bass Loop Cube being manipulated by user

Fig. 1: Comparison of interface sections

The GUM requires docking of a loop cube to allow audio playback via buttons. A Loop Cube (LC) is an object for placement within a GUM; each face of the LC has unique visual representation that pertains to a loop of music. Sphere objects control binaural panning and distance based volume attenuation of their track given position in 2D (XZ) space. Prism objects are parameter space widgets for controlling track effects, mapping is unique to each object. Mapping strategies are in two forms: scaled mapping of object position to Ableton Macro controls and interpolated parameter spaces utilising Manifold-Interface Amplitude Panning 31. Track effects include: (i) Drum - Interpolated convolution reverb amount and type. 3D (XYZ) VR space to 2D (XZ) effect mapping. The further away the object the wetter the signal mixture. Position in the VR space blends size of reverb, going clockwise: front-right $=$ cavernous, back-right $=$ large hall, back-left $=$ room, front-left $=$ small tight space. (ii) Bass - Filter cut-off, 3D (XYZ) VR space to 1D effect mapping. Object distance from user sets frequency, further from user sets higher frequency. (iii) Synth - Duration and timbre of notes. 3D (XYZ) VR space to many dimensional mapping with complex relationship of spatial parameter to sonic output. Approximately, distance changes length of notes and height alters timbre.

Interaction features Two distinct interface metaphors are present in the artefact: (a) Docking metaphor where users must place objects within other structures to yield further functionality, (b) Spatial interaction metaphor, objects control audio modulation parameters based on mappings of hand and head movement in the space.

Various interaction dynamics are present to improve usability and aesthetic enjoyment: (i) Magnetic objects and grabbing gestures to expand interaction space on top of direct object contact. Objects are selected and hyper-realistically 25 grabbed using iconic pinch or grab gestures that only select objects available within a certain bounding box based on the visual field (see figure 2). Expanded depth of interaction space is gained at the cost of users having to learn behaviours 


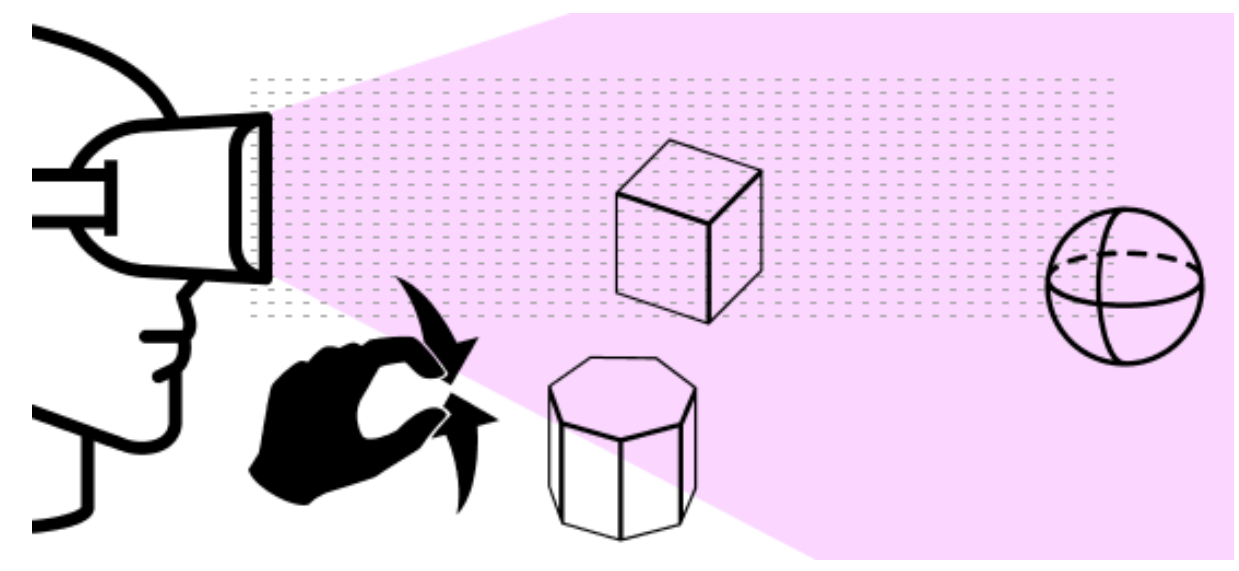

Fig. 2: Magnetic grabbing gesture and bounding rules. Hatched area indicates selectable objects boundary. Closest object will be grabbed, in this case the cube not the heptagon. Shaded area is approximate HMD field of vision.

and their issues; (ii) Gaze-based movement of GUMs was implemented to have them move back and forward only when looked at; (iii) Object-based gravity was implemented to ameliorate interaction errors, where if an object is flung beyond the range of direct control a force is applied to the object to always bring it back to within grabbing range; (iv) A 3D matrix of points appears on grabbing a prism, this indicates the boundaries of possible spatial interaction; (v) Light-streams indicate the object that is currently closest and available for grabbing, figure $1 \mathrm{~b}$

Though challenging in music interfaces, audio feedback of action is implemented for: (i) Loop Cube (LC) grabs, using single note of $\mathrm{C}$ minor triad with note being mapped to object group, Drums = C3, Synth = D\#3, Bass = G3; (ii) Successful docking of a LC in a Grid Unit Menu (GUM); (iii) Audio and visual explosion feedback occurs when a Sphere or Prism makes contact with the centre of the GUM, indicating that it does not belong there, figure $1 \mathrm{a}$.

Incremental introduction of environment objects is utilised so that functionality is explored one piece at a time. At the start of the experience only one LC is presented to user while other objects are hidden, once this object has been grasped another object will appear, this process continues one by one till all objects are available.

\section{User Evaluation}

The evaluation of the system uses a mixed methods approach based on semistructured interviews, surveys and video-based interaction analysis. The methodology evaluates experience, interaction, and knowledge representation, by triangulated analysis of multimodal data. 


\subsection{Participants and Setting}

Twenty-three participants (9 female, average age 28) were recruited from Queen Mary University of London email lists and special interest groups (music, technology, VR) from meetup.com. Demographic data was collected alongside Likertbased self-assessments. Novice and Expert groups were based on response to the self-assessment item ${ }^{3}$ I am experienced at using music software and sound synthesis. The response scale had 5 points pertaining to experience level (Not at all - Very much) points $1 \& 2$ are aggregated as Novice $(\mathrm{N}=8)$ and points $4 \& 5$ were grouped as Expert $(\mathrm{N}=9)$; with point 3 being assigned as Moderate $(\mathrm{N}=6)$.

All sessions and interviews were conducted in the Media and Arts Technology Performance Lab in Queen Mary University of London. Study was carried out following ethical review by the Queen Mary Ethics Committee (Approval ID QMREC1522a), all participants provided informed consent.

\subsection{Experimental Procedure}

Participants engaged in two uses of Objects VR, the Explore session and the Task session. Interviews were conducted after each session and survey metrics were completed after the second interview. The testing procedure was adapted from [34, where process of data collection was purposeful to obtain the most subjective representations of experience before questionnaires were issued that may introduce bias to self-report. In the Explore session no training or indication of function was provided before the session, and the concept of a musical loop and effects controllers was not introduced. Participants were told to just play with the interface and see what they could learn and how they felt using it. The Task session came after a functionality briefing about the environment dynamics and interaction gestures, but no details of sonic or musical function were divulged. The task of that session was to use the interface again to accomplish more musical ideas. No formal performance goals were set, the task was to just make music. The experiment had the following procedure, average or approximate times per section indicated:

1. Explore Session $(M(S D)=8 \min 30 \mathrm{~s}(1 \min 26 \mathrm{~s}))$;

2. Interview A $(M=6 \min 02 s$ (2min 25s));

3. Functionality Briefing (3-5 min);

4. Task Session $(M=6 \min 10 \mathrm{~s}(3 \mathrm{~s}))$;

5. Interview B $(M=6 \min 38 s$ (2min 11s));

6. Surveys (5-15 $\mathrm{min})$.

As of incremental learning rules the Explore session had a longer session time. Overall the whole process took between 40-45 minutes.

To locate meaning within the qualitative data, inductive thematic analysis was used based on Braun and Clarke's six step process: familiarise with the data,

\footnotetext{
${ }^{3}$ Other groups self-assessment items were included for VR experience and interactive sensor experience, but group numbers of novice and expert were highly skewed so are not evaluated here.
} 
generate initial codes, search for themes, review themes, define and name themes, and produce a report 9 . In an inductive revision and organisation of codes, categories and themes emerge from the data. Coding of VR interface interactions is based on the annotation of phases of action using Interaction Analysis (IA) [23. IA offers a structured approach that has been used previously for analysing musical interaction [5]. IA de-marks ordered units of activity, signified by: shifts in activity, shifts of attention, use of spatial alignment and posture, spatial organisation of activity. Useful categories for bounding actions include observation of patterns such as: how sections of action begin and end, levels of repetition and variability of actions, entry and exit strategies of interaction.

\section{Qualitative Results and Analysis}

\subsection{Behavioural data}

Data included video of HMD screen-grab of VR interactions, two video perspectives of body actions in the physical testing space, audio recordings of a voice mic and the music created, and video of interviews. To work with the multimodal data, content was arranged for combined analysis using Final Cut Pro 10 to synchronise and layer all data sources. This combined perspective data was then imported into MAXQDA 12 for analysis, where VR and physical interactions were evaluated using IA and interview self-report was analysed using thematic analysis.

The following notation is used to indicate what data sources a summary of occurrences or quote came from. Grab(x) means code "Grab" was observed x times. $(\mathrm{Px})$ : participant number e.g. (P7) is participant seven; $(\mathrm{N}=\mathrm{x})$ : participant occurrences in a theme or explanation. In theme summaries instances of observation are counted once per participant e.g. Play(6) six different users refer to theme Play. Moderates were excluded from qualitative analysis due to insufficient group numbers and the focus on comparing novice and expert results.

\subsection{Explore Session Interaction Analysis}

Through IA coding of the Explore session a coding system was developed. Relevant codes are included in table 1 .

Interaction Analysis Themes The following analysis highlights features of user interaction with Objects $V R$ in the explore session before interview A (users' first contact with the interface). Vignettes and codelines were used to exemplify common or interesting cases, as for many issues a deep level of detail is needed to unpack user behaviour.

Sticky Objects Due to magnetic grabbing implementation not working well, many users struggled with objects attaching themselves to their hands. Marked by code Sticky Object, and frequently with Unexpected $>$ CoF. Sticky objects had the 
Table 1: Subset of Interaction Analysis code system from Explore session

\begin{tabular}{|c|c|c|}
\hline \multicolumn{3}{|c|}{ Operational(1534): Itemised interactions with the interface. } \\
\hline $\operatorname{Code}(\mathrm{n})$ & Group & Brief Description \\
\hline Button Success $(279)$ & Actions & User presses button on purpose \\
\hline Grab LC (143) & Actions & User grabs loop cube \\
\hline Put LC (68) & Actions & User places loop cube in GUM \\
\hline Prism Feedback (50) & Actions & Prism enters GUM space \\
\hline Sphere Feedback (25) & Actions & Sphere enters GUM space \\
\hline Eject LC (21) & Actions & Purposeful ejection of LC from GUM \\
\hline Sticky Object (114) & Problems & see Sticky Objects theme \\
\hline Button Error (111) & Problems & Unsuccessful button press, or accidental press \\
\hline Grab Error (61) & Problems & Unsuccessful grab action \\
\hline Utterance $(87)$ & Interactions & Statement or vocal sound \\
\hline Posture(20) & Interactions & Embodied interaction to stimulus \\
\hline Gesture(18) & Interactions & Interesting use of hands and arms \\
\hline \multicolumn{3}{|c|}{ Interpretive(262): Theories around micro-phases of user action. } \\
\hline $\operatorname{Code}(n)$ & Group & Brief Description \\
\hline Change of focus (31) & Attention & Natural change in action pattern \\
\hline $\begin{array}{l}\text { Unexpected > CoF } \\
(34)\end{array}$ & Attention & Surprise causing change in action patterns \\
\hline $\begin{array}{l}\text { Observe after action } \\
(21)\end{array}$ & Attention & Pausing after action or feedback \\
\hline $\begin{array}{l}\text { Probing for } \\
\text { functionality (37) }\end{array}$ & Physical & $\begin{array}{l}\text { Systematic pattern of actions with interface } \\
\text { element }\end{array}$ \\
\hline Discovery (37) & Conceptual & $\begin{array}{l}\text { First time feedback or function witnessed, often } \\
\text { with embodied reaction }\end{array}$ \\
\hline \multicolumn{3}{|c|}{ Strategies(106): Assumptions about macro-phases of user actions } \\
\hline $\operatorname{Code}(\mathrm{n})$ & \multicolumn{2}{|c|}{ Brief Description } \\
\hline Exploring (39) & \multicolumn{2}{|c|}{ Unstructured phases of interaction } \\
\hline Experimenting (54) & \multicolumn{2}{|c|}{ Systematic or repetitive phases of interaction } \\
\hline
\end{tabular}


impression of being quite frustrating, marked by distressed or volatile release attempts and various utterances. But by interrupting users focus sticky objects maybe allowed opportunities for learning. Examples of such issues are detailed in table 2 and are described further in the theme Surprises.

Repetitive Actions The Probing for functionality and Experimenting codes annotated inferences related to determining functionality often based on repetitive actions. Common patterns included: filling GUMs with each different type of object one after another, systematic button pressing either individually or in dual button holds (figure 3), and trying to join cubes together. The actual goal of such experimentation was subjective and better left to triangulated analysis.

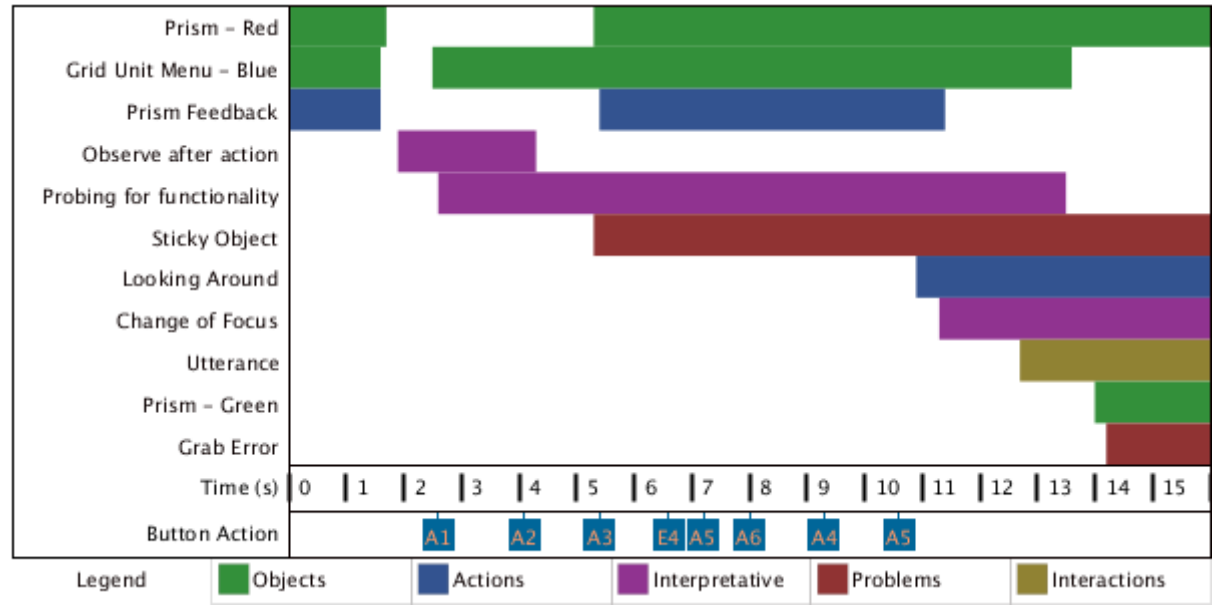

Fig. 3: Novice P3 Probing for Functionality. Systematic button pressing without audio playback (no LC in GUM).

Audio Feedback When trialling elements to determine functionality, as in Repetitive Actions, many objects only provided musical feedback when the track was running, this may lead to incorrect assumptions of system function as of ambiguous feedback. It is important to distinguish without audio and with audio probing. A comparison is made between P3 in figure 3 and P4 in table 2. In the without audio playback case, P3 quickly moves through GUM buttons, finds little happening, and moves to exploring other objects. For the with audio case, a different pattern of probing was seen; P4 switched on and off the buttons with associated musical loops switching on and off. Pattern of action is broken by a Sticky Object attaching to them.

Many users experienced the prisms and spheres as feedback objects (Prism Feedback and Sphere Feedback) either accidentally or on purpose. While the 
Table 2: Novice P4 Probing for Functionality with audio playback and Sticky Objects

\begin{tabular}{c|l}
\hline Timecode & Sequence of action (Interpretations) \\
\hline 00:07:30 & Trialling buttons: switching on and off, repeats for different buttons \\
00:07:46 & Sticky green prism attaches to hand -> Change of focus to green prism \\
00:07:51 & $\begin{array}{l}\text { Ejects LC from green GUM using button while holding prism }->\text { Tries } \\
\text { to put prism into GUM, causing explosion Feedback -> P4 pauses for 1 } \\
\text { second }\end{array}$ \\
00:08:00 & $\begin{array}{l}\text { Sticky blue bass prism attaches to hand, P4 changes focus to it }-> \\
\text { Movement yields new audio feedback in the form of distinct filter } \\
\text { parameter modulation }->\text { P4 uses blue prism for 35s }\end{array}$ \\
\hline
\end{tabular}

feedback does provide adequate indication of negative operation, and stimulating audio-visual effects, if the track is not active its musical function may not be determined. As an example, during a phase of probing, P1 was holding a sphere and error feedback effects were caused while trying to use buttons. From the video it appears as if $\mathrm{P} 1$ was more interested in determining the function of the buttons, and then feedback noise happens. Potentially, this sequence of actions confused their mental model of function, as reliable links between actions, reaction and stimulus were not clearly made. The user was left wondering whether sound is present because they pressed a button or because they put the ball in the box. Anecdotally, P1 reported negatively on many items in the sonic interaction questionnaire and interview self-report was predominantly around negative themes.

Surprises Relating to the previous theme of Sticky Objects, the Surprises theme marks occurrences of possible learning from system error, often annotated by the Discovery code. Discovery intersected with the Utterance code 11 times, where an 'out-loud' signalled their reaction to the stimulus. An out-loud can be considered to render 'private' activity 'public' and visible 19]. Through action, elements interplay may cause realisation of function or new possibility to users, who make visible their reaction. Also, noticeable focus shifts and altered interaction phases potentially mark a discovery. The link of verbal utterance, gestures and posture in isolating possible moments of discovery proved a useful technique. An example of an accident leading to interface exploration can be seen in table 2, at time-code 00:08:00 where a Sticky Object bass prism attaches to their hand while audio is playing and changes interaction pattern for significant period of time.

\subsection{Explore Session Interview Themes}

Analysis of the Explore session interviews highlighted a series themes within self-reports across Novice and Expert groups. High-level themes include Comprehension, Confusion, Engagement, Frustration, Interface, Interaction, Altered states of perception (ASoP), Goals and models (GaM). 
Thematic analysis results are separated into Novice (table 33), Expert (table 4) and Shared (table 5) themes.

Table 3: Novice Interview Analysis Themes

\begin{tabular}{|c|c|c|}
\hline Theme (n) & Description & Theme relations \\
\hline $\begin{array}{l}\text { Varied } \\
\text { conceptual } \\
\text { models }(7)\end{array}$ & $\begin{array}{l}\text { Highly varied interpretations of system as } \\
\text { their conceptual model. }\end{array}$ & $\begin{array}{l}\text { Comprehension, } \\
\text { Confusion, GaM }\end{array}$ \\
\hline $\begin{array}{l}\text { Interface } \\
\text { Feedback (5) }\end{array}$ & $\begin{array}{l}\text { Objects fed back subjectively useful } \\
\text { information about what was possible. }\end{array}$ & Comprehension, GaM \\
\hline $\begin{array}{l}\text { Colours and } \\
\text { Groups (4) }\end{array}$ & $\begin{array}{l}\text { Visual relationships made sense, but not as } \\
\text { track or audio function. }\end{array}$ & $\begin{array}{l}\text { Comprehension, } \\
\text { Interface }\end{array}$ \\
\hline $\begin{array}{l}\text { Empowered } \\
\text { Interaction (4) }\end{array}$ & $\begin{array}{l}\text { Control and interaction felt "physical", } \\
\text { "immersive", "powerful", "magical". }\end{array}$ & $\begin{array}{l}\text { Engagement, } \\
\text { Interaction }\end{array}$ \\
\hline $\begin{array}{l}\text { Not getting it } \\
(4)\end{array}$ & $\begin{array}{l}\text { Feeling of missing some level of } \\
\text { understanding that was annoying. }\end{array}$ & Frustration, Interface \\
\hline Objects (4) & $\begin{array}{l}\text { Uncertainty of what objects did and their } \\
\text { relationships. }\end{array}$ & Confusion, GaM \\
\hline Body (3) & $\begin{array}{l}\text { Experience of altered perception of self or } \\
\text { perceptual incongruence: dream-like; feeling } \\
\text { of not having a body; floating and weird. }\end{array}$ & $\begin{array}{l}\text { ASoP, Enjoyable, } \\
\text { Strange but I like it }\end{array}$ \\
\hline $\begin{array}{l}\text { Hearing } \\
\text { Differences (3) }\end{array}$ & Confusion in distinguishing musical changes. & Confusion \\
\hline $\begin{array}{l}\text { Playful } \\
\text { exploration (3) }\end{array}$ & $\begin{array}{l}\text { Description of playful discovery, exploration } \\
\text { and reward. }\end{array}$ & $\begin{array}{l}\text { GaM, Self-learning, } \\
\text { Playing }\end{array}$ \\
\hline Purpose (3) & $\begin{array}{l}\text { Feeling "lost" or uncertain in general of } \\
\text { what to do. }\end{array}$ & Confusion, GaM \\
\hline $\begin{array}{l}\text { Learning Issues } \\
(2)\end{array}$ & $\begin{array}{l}\text { Taking a long time to learn gestures and how } \\
\text { to interact. }\end{array}$ & $\begin{array}{l}\text { Frustration, } \\
\text { Interaction }\end{array}$ \\
\hline
\end{tabular}

An important theme that disambiguates Novice from Expert users is Comprehension. The theme marks self-report of understanding components of their experience and the interface. The Confusion theme describes how levels of uncertainty manifest in self-report. A common theme with high levels of uncertainty in interface and interaction in general, but the content of what was confusing varies across groups. The Engagement theme draws attention to highly varied representations of what was engaging for individuals in the experience with many shared components across groups. The only aberrant case is Novice P1 who experienced only Confusion and Frustration. The Frustration theme marks self-report around levels of frustration with their experience. All users struggled with gestural interaction, interaction dynamics and the way objects 
Table 4: Expert Interview Analysis Themes

\begin{tabular}{|c|c|c|}
\hline Theme (n) & Description & Theme relations \\
\hline $\begin{array}{l}\text { Functional } \\
\text { Understanding } \\
\text { (9) }\end{array}$ & $\begin{array}{l}\text { Detailed description of system, with links to } \\
\text { audio functionality. }\end{array}$ & $\begin{array}{l}\text { Comprehension, } \\
\text { Confusion, Interface }\end{array}$ \\
\hline $\begin{array}{l}\text { Colour and } \\
\text { Sound (8) }\end{array}$ & $\begin{array}{l}\text { An understanding of colour and shape } \\
\text { relationships to tracks. }\end{array}$ & $\begin{array}{l}\text { Comprehension, } \\
\text { Interface }\end{array}$ \\
\hline $\begin{array}{l}\text { Frustrated } \\
\text { Interaction (6) }\end{array}$ & $\begin{array}{l}\text { Frustration with interaction at lack of } \\
\text { adequate control. }\end{array}$ & $\begin{array}{l}\text { Frustration, } \\
\text { Interaction }\end{array}$ \\
\hline Intention (6) & Their goal was to make music & GaM. \\
\hline Novel (4) & $\begin{array}{l}\text { Descriptions of the novelty of musical } \\
\text { interface and experience as enjoyable; } \\
\text { despite frustration and uncertainty. }\end{array}$ & Engagement, GaM \\
\hline Metaphors (2) & $\begin{array}{l}\text { An awareness of interface metaphors in } \\
\text { self-report. }\end{array}$ & $\begin{array}{l}\text { Comprehension, } \\
\text { Interaction }\end{array}$ \\
\hline
\end{tabular}

worked hence this theme couples extensively with the Interaction theme in both groups. How and to what level frustration was reported indicates slight difference in emphasis between groups.

The Interface theme relates to the functionality of the environment objects. Analysis is grouped by the functionality category: Loop playback objects (GUMs, LCs and Buttons) or effects control objects (Spheres and Prisms). The Interaction theme relates to control, gestural interaction, and interface dynamics. It highlights some important differences in Novice and Expert participants. Novices talked more positively about their feeling of interaction and control than the Expert group, though still citing similar errors such as Sticky Objects. Whereas the Expert group highlighted their interaction with mostly frustration at the lack of adequate control. The Altered states of perception theme was often related to the Engagement theme and may link to flow and spatial presence theories. Novice altered states were described as enjoyable and bodily oriented. In contrast, the Expert group described altered states of perception with less of an emphasis on a relationship to their bodies, instead report related to task immersion in music but with equally evocative terms. The Goals and models theme highlights how participants conceptualised the system and their purpose in using it, rather than discrete levels of system function.

\subsection{Task Session Interview and Interaction Analysis}

Similar patterns of activity and interview topics were observed in the Task session. Key themes of difference, reflection and change are presented in table 6 (Users with moderate experience were excluded from the analysis). Of the persistent issues that occurred, Novices had continued problems with Hearing Differences in musical content, and general confusion over the conceptual model of function. Both groups continued to report uncertainty around what the spheres 
Table 5: Shared Interview Analysis Themes

\begin{tabular}{|c|c|c|}
\hline Theme & Description & $\begin{array}{l}\text { Group and Theme } \\
\text { relations }\end{array}$ \\
\hline Enjoyable (22) & $\begin{array}{l}\text { Used of positive vocabulary to report } \\
\text { aspects of experience, across all but one } \\
\text { participant (P1). }\end{array}$ & $\begin{array}{l}\text { Engagement, ASoP, } \\
\text { Strange but I like it }\end{array}$ \\
\hline $\begin{array}{l}\text { Strange but I } \\
\text { like it (10) }\end{array}$ & $\begin{array}{l}\text { Use of "Strange", "weird" or "odd" to } \\
\text { describe experience but the clarifying the } \\
\text { term as enjoyable. }\end{array}$ & $\begin{array}{l}\text { Engagement, ASoP, } \\
\text { Enjoyable }\end{array}$ \\
\hline $\begin{array}{l}\text { Self learning } \\
(9)\end{array}$ & $\begin{array}{l}\text { Report of self-learning through interface } \\
\text { affordances and feedback. Includes } \\
\text { encouragement to learn, moments of } \\
\text { discovery, and "intuitive" interface } \\
\text { dynamics. }\end{array}$ & $\begin{array}{l}\text { Engagement, GaM; } \\
\text { Novice: Playful } \\
\text { Exploration; Expert: } \\
\text { Immersed in sonic } \\
\text { interaction }\end{array}$ \\
\hline $\begin{array}{l}\text { Loop playback } \\
\text { objects }(8)\end{array}$ & $\begin{array}{l}\text { Understanding of functionality varies by } \\
\text { group. }\end{array}$ & $\begin{array}{l}\text { Interface; Novice: } \\
\text { Confusion; Expert: } \\
\text { Comprehension; }\end{array}$ \\
\hline $\begin{array}{l}\text { Effects objects } \\
(7)\end{array}$ & Prisms and spheres were confusing. & Confusion, Interface \\
\hline Play (6) & Interaction was "playful" or "playing". & Engagement, GaM \\
\hline $\begin{array}{l}\text { Immersed in } \\
\text { sonic } \\
\text { interaction (5) }\end{array}$ & $\begin{array}{l}\text { Description of being immersed in sonic } \\
\text { interaction as creative musical } \\
\text { engagement, task immersion or feelings of } \\
\text { connection to sound. }\end{array}$ & $\begin{array}{l}\text { ASoP; Novice: } \\
\text { Engagement; Expert: } \\
\text { Engagement, GaM }\end{array}$ \\
\hline
\end{tabular}

purpose was, and in one anomaly an Expert user forgot sphere functionality across sessions. There was continued errors around implementation and sensor issues, such as Sticky Objects. The Improved experience theme collects instances of more effective probing and gestural control seen in Task session, except for two aberrant cases: (i) gestural control was worse (P19), (ii) did not play any audio using GUMs in second session (P2). Barring these exceptions, instances of enjoyable experience with music and interaction were reported. The following sub-themes highlight improvements:

- Being more aware of surroundings: Users interacted with more objects, possibility due to functionality briefing indicating that other objects existed;

- More focused(5): Self-report of being more focused on getting an understanding to make objects to do something they wanted.

\section{Quantitative Results and Statistical Analysis}

Quantitative data relating to user experience was obtained through a series of post-experience questionnaires, where responses relate to the whole experience. These are: (i) Sonic Interaction Design (SID) questionnaire a five-point Likert 
Table 6: Task Session Thematic Analysis

\begin{tabular}{l|l|l}
\hline Theme & Description & Group \\
\hline $\begin{array}{l}\text { Changing } \\
\text { strategies (8) }\end{array}$ & $\begin{array}{l}\text { Early in the Task session there are more instances of } \\
\text { direct and immediate sound actions with LC and GUM } \\
\text { elements to trigger sounds, compared with their previous } \\
\text { interaction in the Explore session. }\end{array}$ & Shared \\
$\begin{array}{l}\text { Less frustrating } \\
\text { Instances of more confident gestures and sets of actions. } \\
\text { Self-report of still being difficult to interact, but better in } \\
\text { general. }\end{array}$ & Shared \\
$\begin{array}{l}\text { More sonic } \\
\text { control (5) }\end{array}$ & $\begin{array}{l}\text { IA observation of improved control and self-report of } \\
\text { feeling more in control. }\end{array}$ & Novice \\
$\begin{array}{l}\text { Need more time } \\
\text { Persistent levels of confusion, indicting that they needed } \\
\text { more time } \\
\text { Difficulty of reproducing sonic effect with prism } \\
\text { mappings, self-report attributes parameter mappings as } \\
\text { quite unclear, though they understood overall function. } \\
\text { mappings (3) }\end{array}$ & Shared \\
$\begin{array}{l}\text { Plateau of } \\
\text { possibilities (3) }\end{array}$ & $\begin{array}{l}\text { Expert } \\
\text { extended use. }\end{array}$ & Shared \\
\hline
\end{tabular}

assessment of flow and sonic interaction, designed for the study based on items from [5. SID item statements are presented with their results in section 6.1 (ii) Creativity Support Index [11] (CSI) was used to determine the value of the interface as a creativity support tool (CST) for music. CSI item statements can be found in publication [11]; (iii) Spatial Presence Experience Scale [18](SPES) was used as a presence metric that integrates concepts of spatial presence 37, it functions as a diverse media measure of presence. SPES item statements can be found in 18 .

Moderate group results were used within statistical analysis, but most comparisons were conducted to look at the difference between Novice and Expert groups.

\subsection{Sonic Interaction Questionnaire Results}

The median (Mdn) and interquartile range (IQR) for all Sonic Interaction (SID) items can be seen in figure 4 .

- SID.1 I was connected to the sound changes through my actions A Mann-Whitney's U (MWU) test found a near significant result for independent factor Group when comparing Novice and Expert, $U=19.5, Z=$ $-1.72, p=0.09, r=0.36$. Despite the lack of significant difference between groups, the general trend is of strong agreement that users had agency over sound. This puts emphasis on the interview analysis to determine what level of sonic agency was experienced.

- SID.2 I could relate my actions to specific sonic effects A KruskalWallis $\mathrm{H}(\mathrm{KWH})$ test revealed a near significant effect of Group on SID.2 


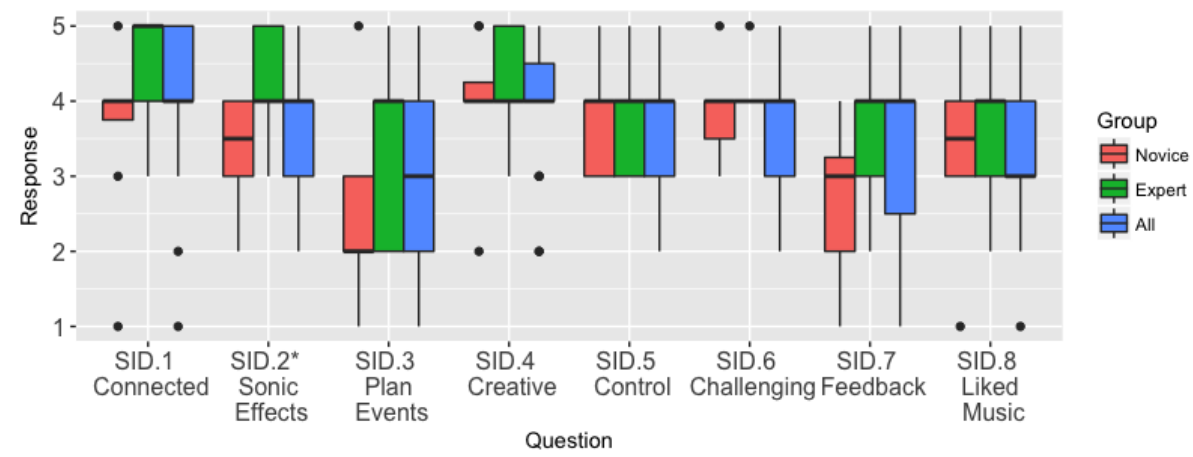

Fig. 4: Sonic Interaction Questionnaire box and whisker plots. Asterisk marks items with significant differences. $\mathrm{Y}$ axis response scale values: $1=\mathrm{I}$ do not agree at all; $2=\mathrm{I}$ do not agree; $3=$ Neither Agree or Disagree; $4=\mathrm{I}$ agree; 5 $=\mathrm{I}$ fully agree.

response $\left(\chi^{2}(2)=4.81, p<0.09\right)$. A MWU test and found a significant difference between Novices and Experts $(U=15.5, Z=-2.16, p<0.05, r=$ 0.45 ), with the mean ranks of Novices and Experts being 3.38 and 4.22 . While both groups overlap between neutral and agreement, the expert group had a more positive tendency to relate action to sonic effects. This finding could corroborate the thematic analysis theme of Hearing Differences, indicating that Experts and Novices appreciation of action and sonic effect was not observed to be the same in this study.

- SID.3 I could plan sonic events using objects No significant result was found comparing all groups (KWH test) or comparing Novices and Experts (MWU test). The results average around neutral but with groups having differing trends, Novices $(M=2.5(1.2))$ tend to disagree more than Experts $(M=3.44(1.13))$ that they could plan sonic events.

- SID.4 I felt part of a creative process The dominant trend was that all users agreed that they felt part of a creative process.

- SID.5 I felt in control Positive result where responses tended towards neutral and agreement.

- SID.6 It was challenging Novice and Expert responses tended to agree that they found the process challenging. To link to flow conceptualisations about challenge and reward, a more detailed analysis of what was challenging is required.

- SID.7 The feedback in the system made sense Item had a netural tendency with no significant difference found for comparing all groups (KWH test) or comparing Novices and Experts using a MWU test, despite different distributions of positive and negative tendencies.

- SID.8 I liked the music I created Given the fixed nature of the musical content the neutral to agreement responses are positive. This item requires further testing in different genres of music to determine whether stylistic boundaries alter reaction to the interface. 


\subsection{Spatial Presence Experience Scale Results}

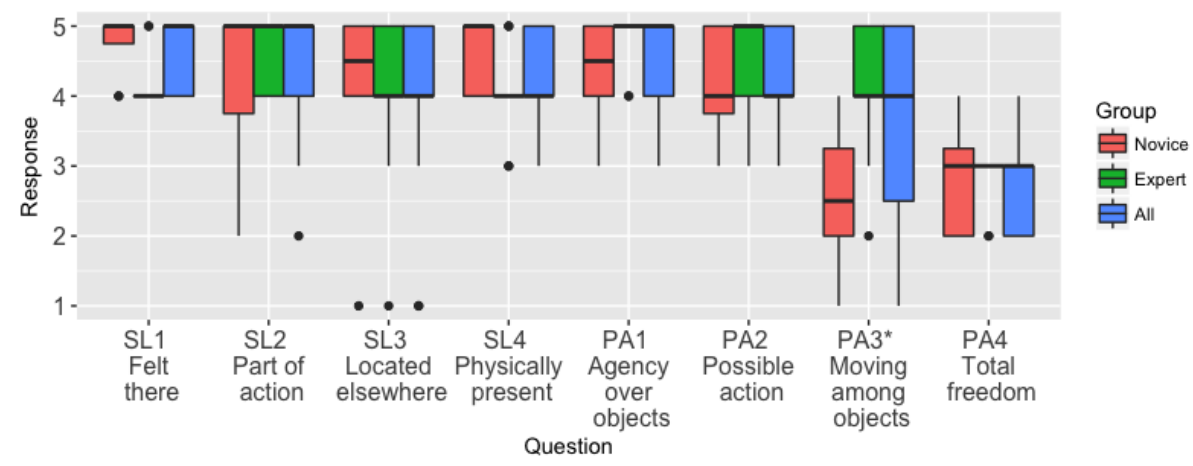

Fig. 5: Spatial Presence Experience Scale box and whisker plots. Y axis is response scales same as figure 4 .

Self-location (SL) items There is strong agreement that participants felt "there" in the virtual environment (SL1), and were actively taking part in action (SL2), and that they felt like they were located in a different environment from the physical testing area (SL3). Also results indicate predominantly agreement for feeling physically present in interface environment (SL4). No significant difference for group was observed across SL items.

Possible actions (PA) items There is strong agreement that participants felt they had agency over objects in the environment (PA1), and agreement that there was possibility for action within the environment (PA2). There is a significant difference for group in responses to 'feeling like they could move among the objects in the environment' (PA3) $\left(\chi^{2}(2)=6.76, p<.05\right)$. MWU pairwise comparisons using Holm-Bonferroni adjustments indicate a moderately significant effect of group Novice $(M=2.63(1.06))$ and Expert $(M=4.11(1.05))$ $(U=11.5, Z=-2.42, p<.05, r=0.51)$. So Novices felt less possibility to move throughout objects in the virtual environment than Experts. On average participants were neutral about having total freedom to do whatever they wanted in VR (PA4) with no significant differences present across groups.

\subsection{Creativity Support Index Results}

The CSI is a psychometric survey designed to evaluate a Creativity Support Tool (CST). It measures user, tool and task by weighting factors to their importance in the task. Factors evaluated include: Exploration, Expressiveness, 
Table 7: Table of CSI Factor Comparisons

\begin{tabular}{|c|c|c|c|}
\hline & $\begin{array}{l}\text { Average } \\
\text { Factor } \\
\text { Count }\end{array}$ & $\begin{array}{l}\text { Average } \\
\text { Factor } \\
\text { Score }\end{array}$ & $\begin{array}{l}\text { Weighted } \\
\text { Average } \\
\text { Factor Score }\end{array}$ \\
\hline Factor & $\operatorname{Mdn}(\mathrm{IQR})$ & $\mathrm{M}(\mathrm{SD})$ & $\mathrm{M}(\mathrm{SD})$ \\
\hline Enjoyment & $3(2-4)$ & $8.13(1.52)$ & $48.61(23.16)$ \\
\hline Exploration & $4(2-4)$ & $5.98(2.05)$ & $40.7(19.75)$ \\
\hline Expressiveness & $3(3-5)$ & $5.7(2.08)$ & $41.48(22.74)$ \\
\hline Immersion & $3(2.5-4)$ & $7.3(2.23)$ & $46.96(28.01)$ \\
\hline Results Worth Effort & $1(1-2)$ & $6.76(1.76)$ & $20.22(19.05)$ \\
\hline
\end{tabular}

Immersion, Enjoyment, Results Worth Effort (RWE), and Collaboration ${ }^{4}$ CSI results contains the following features:

- Average Factor Count (AFC) indicates what is important to users in a creative task. Counts are based on 15 pair-wise comparisons of CSI factors. AFC therefore scores task importance and weights the average factor scores and final CSI score.

- Average Factor Score (AFS) is the average responses to two Likert scale questions for each factor. It indicates the direct response of the user to the tool for the task to that factor.

- Weighted Average Factor Score (WAFS) is sensitive to the relative importance of factors reflecting the relationship of tool, task and user.

- Overall CSI score is an index score out of 100, where a higher score indicates better creativity support.

Results summaries See Table 7 and Figure 6 for factor result:5

- CSI scores The general tendency of the population for the combined CSI scores place ObjectsVR in a positive light with a high average score $(M=$ 65.94(14.45)), no significant effects were observed based on Group and distributions were similar. Compared to other CSI scores in literature this is good result 3, 21, 36 and is a useful benchmark for future iterations.

- Enjoyment The WAFS for the enjoyment factor was the highest average across the whole population; with no significant differences present across groups. Enjoyment is a moderately important task factor given AFC.

\footnotetext{
${ }^{4}$ Although collaboration is not a feature of Objects $V R$, the factor must be kept to preserve the CSI scoring structure.

${ }^{5}$ Lack of normality in the data means non-parametric statistics were chosen for all tests of significance. It was considered to transform the data, but the sample sizes per group in the sample would question the validity of any results. Collaboration factor is not discussed.
} 


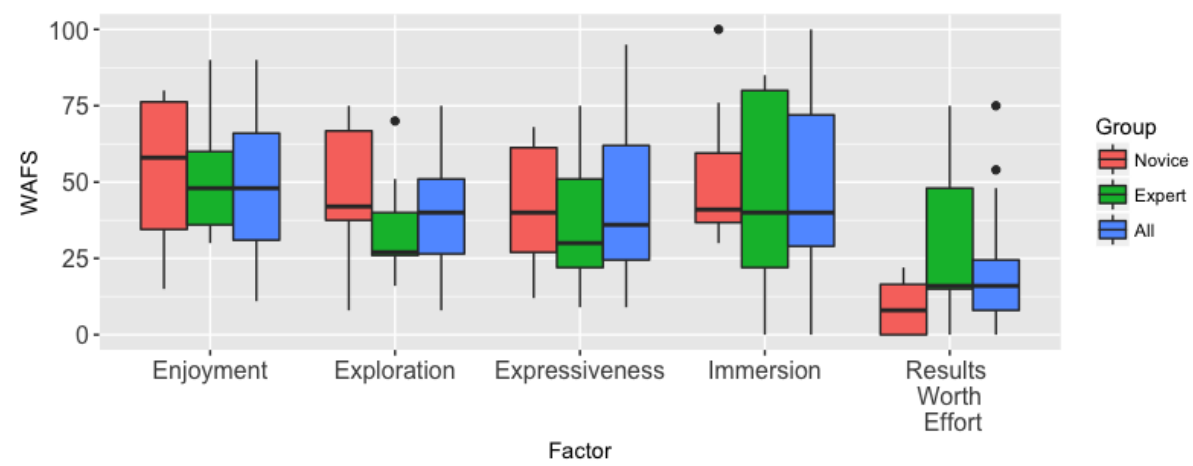

Fig. 6: Creativity Support Index box and whisker plots of Weighted Average Factor Scores.

- Exploration AFC indicates exploration of ideas and possibilities is an important feature in a making music task. No significant results were observed between groups. The WAFS for Exploration was higher for the Novice group than the Experts (table 6), but with no significant difference.

- Expressiveness The AFS for expressiveness of the tool scored average to well across the whole population. WAFS show a moderate tendency across the sample with no significant results for group.

- Immersion average values for AFC highlight Immersion with moderate importance (table 7), but as with expressiveness the variance of the Expert results are of interest $(M=3.0(1.36) ; M d n=4 ; I Q R: 2-4$, Min $=0$, Max $=$ $5)$. Moderately important AFC with high AFS produced a WAFS of moderate importance, but with high variance; with no significant differences were observed for groups.

- Results Worth Effort Compared to the relatively low AFC, the RWE AFS was quite positive (table 7). The WAFS exhibited a near significant result for group (KWH test: $\left.\chi^{2}(2)=4.19, p=0.12\right)$. A MWU test of Novice and Expert groups was not significant $(U=18, Z=-1.74, p=0.09, r=0.36)$, but again near significant. The WAFS has comparatively low average to other factors.

\section{Discussion}

We discuss in this section of how well the Objects $V R$ artefact satisfied its original design criteria. By reflecting on results and their means of observation, we can evaluate our design method to determine implications for the design and research of interactive music systems in VR. Additionally, the analysis and advice offered is case-based and should be understood to be provisional and exploratory.

\subsection{Design criteria assessment}

Accessible and fun for novices. Despite the brief time in the environment across both sessions, novices tended to find it an engaging and enjoyable experience 
of interacting with musical content, barring aberrant cases. Self-report marked high levels of enjoyment and engagement (Enjoyable, Playful, Strange but I like it) despite difficulties of interaction. The IA marked noticeable improvement in interface use across both sessions (More sonic control). The experience was predominantly considered to make them feel creative (SID.4) and the highest WAFS from the CSI was Enjoyment. But, high levels of confusion and frustration were observed in self-report (Not getting it, Objects, Hearing differences) with IA themes marking persistent issues with interaction (Sticky Objects, Button Error, Grab Error). Findings offer many implications for design improvement to enhance creative engagement by improving comprehension of musical function (Varied conceptual models) to scaffold understanding of musical function to explore creative goals.

Allow 'physical' experimentation and interaction with musical content. The highest CSI AFC was for Exploration. This highlights that exploring ideas and possibilities is an important feature of a music making task. Positive average results include all user groups feeling "connected to sound changes" (SID.1), being part of a creative process (SID.4), feeling in control (SID.5), and liking the music they made (SID.8). Based on IA, most of the interaction clustered around use of the button-based menus for content playback (Button Success, Grab LC, Put LC, Button Error) to achieve this. When the spatial objects were found they were of interest, and for some the level of feedback produced noticeable changes of focus (Discovery). But comprehension of their function was poor (Objects, Effects objects, Opaque mappings), and usage patterns were often brief and constrained in their use of space. Though in two cases (P4, P23) the movement patterns using prism objects were highly varied, using a large space around them to influence the sound and their interaction. Engagement with a different physical space for making music is still a interesting direction of research to expand the interaction space and achieve the design criteria. But it leaves a challenge: in a medium that affords spatial interaction how do we structure rich and diverse interactions in space with appropriate levels of depth and feedback to the experience?

Obtain high levels of presence. From SPES results, high levels of presence were achieved. Self-report and CSI factor Immersion highlights task immersion as a topic of further investigation at whether presence is correlated in VR interactive music systems. The Immersion factor has a large range across the whole population. Large differences were observed in individual cases for the task of making music. The results range means further analysis is needed to determine how users interpreted the task of making music in a VR environment, to resolve whether the immersive nature of VR is being reported on or if levels of task immersion are achieved for creative tasks. An interesting comparison would be to test similar functionality across media types to determine whether musical tasks are equally as immersive outside of VR.

Allow users to experience flow states. Within self-report there are many examples of musical engagement, but the extensive feature of frustration and confusion 
indicates that potential for flow might be hindered by basic interaction problems. Following is a breakdown of conditions for flow in relationship to this study and artefact.

The initial goal of the first session was exploration and understanding, then music-making is the only goal set for the Task session. So perhaps goals set in the experiment did not provide an optimal condition for flow. Novice self-report highlights some instances feeling lost in initial exploration (Purpose) that could corroborate this assertion. A more directed experiment design that sets direct goals for users using the VR interface may provide a sufficient level of challenge and goals that direct the initial learning of the interface.

The system was considered to have sufficient feedback (SID.7, Interface and Feedback) but the many errors of interaction (Problems and Frustration), incorrect models of function (Varied conceptual models) and misuse of error feedback (Audio Feedback) mean that feedback is unclear. The ambiguity of what feedback applied in exploratory inspection means incorrect assumptions of function could be developed, limiting this condition for flow. Complex and mysterious environments need to contain constructive breakdown of interaction 27], or risk distressing Novices. Environment items should be context-dependent; if a track is not running then it's effectors should not be available. This may help build conceptual models of what can be done but still maintain a playful and magical character to the environment.

The artefact and study determined that users enjoyed their experiences (CSI Enjoyment) while finding it challenging (SID.6). But development of skills in the environment to maintain suitable challenge is potentially limited by a shallowness of system functionality (Plateau of possibilities) and for novices a lack of comprehension to establish confident use. Improvements could be found in less rigid forms of musical content interaction e.g. rather than playing back samples you assemble compositions yourself. But a balance of expressive capability and immediate engagement needs to be addressed. It is feasible that well scaffolded complexity in initial engagement could still allow exploration of rich musical possibilities without too much explicit instruction or knowledge dissemination before use.

One effect of flow is to experience an activity as intrinsically rewarding. Much of the self-report across all groups was about levels of enjoyment and engagement. Linking results from 'feeling part of a creative process' (SID.4), 'liking the music created' (SID.8) and CSI factor RWE; interaction was worthwhile regardless of musical output. This is a positive result for both groups as it positions VR music spaces as engaging experiences. Some assume that Experts desire highly expressive systems [7, but our findings for Expressivity CSI factor suggest a more nuanced understanding. Add to this the near significant results for the RWE factor, and it warrants further understanding of how users frame music making when approaching a novel interactive music system. This prompts questions of whether actionable results are more important than ideation or play, and does the nature of the medium alter expectation in this regard. Maybe Experts judge the medium of the system into what they expect from an experience; they interpret 
VR as non-task oriented and do not impose a need for thinking about "good" results. Given this line of reasoning and RWE AFC finding, it is worth trialling the CSI on more traditional computer music software to determine whether the medium of experience alters results for expert users in similar tasks.

\subsection{Methodology Commentary}

The mixed methods study looked to probe initial behaviours, feelings and thoughts of individuals using Objects VR. This details design issues for new users but also highlights more general design issues around Novice or Expert music systems. The IA and interview themes highlight important features of experience for designing user interaction within the Objects $V R$ design space. The CSI and SID surveys were useful triangulation metrics to explore important concepts around flow and user engagement. But, the disambiguation around sonic and task immersion requires further investigation to look for any differences between Novice and Expert groups in relation to flow in VR interactive music system. The question that grouped novice and expert users, while being imprecise, did provide useful categories for comparison but future studies should use validated measures such as the Goldsmiths Musical Sophistication Index 6 . Also further controls need to be put in place to moderate groups based on VR experience.

\subsection{Design implications for novice VR interactive music systems}

Use natural behaviours Study and implement gestural behaviours that relate to users natural understanding. Multiple selection and manipulation taxonomies should be supported, just as in real life, we can pull, push, shove, or cradle an object. Though this makes creation of environments more difficult, the depth of possible interaction could improve presence, experience and performance.

Design for sensor frailty Sensor field of vision (FOV) and fidelity issues govern design of consumer grade interaction experiences. FOV changes how elements need to be presented in an environment for interaction. In Objects $V R$ magnetic grabs resulted in extensive interaction errors. So, design interfaces to actively understand how and when the FOV is a potential hindrance to action, and design system feedback for user action with the sensor space to learn how to interact optimally.

Expression vs Engagement Balancing a low entry fee with sonic depth is troublesome for music novices, and therefore understanding how a novice approaches and learns is fundamental to designing experience in VR interactive music systems. In Objects $V R$ the audio functionality presented was often too opaque for users to understand, so, keep it simple! The novice theme of Hearing Differences relates to other research on novice music interface design [5,7]; where musical content should be diverse enough to clearly hear the differences of musical contributions.

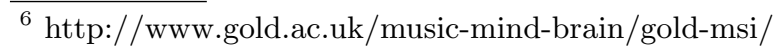


Be careful with divergent metaphors The Objects VR interface utilised multiple interaction metaphors for action-sound relationships. This was confusing to Novices, as signification of function and effect was not clear. We are not advising to avoid using multiple metaphors, rather that clear boundaries, signifiers and transitions are used to guide users through their use.

Create vivid and matching connections Object groups should be visually and behaviourally cohesive to a user. Colours and shapes need to be correctly assigned to allow understanding across the interface. If possible connect features to functionality, but limit overall complexity.

\section{Conclusion}

Designing VR interfaces for creative musical applications provides significant challenges for HCI, requiring a different approach to traditional task-based design of systems. Objects $V R$ is an example of working with the trade-off of expression and engagement to determine the appropriate balance of complexity and expressivity in a virtual reality interactive music system for novice users. It provided most users with an enjoyable experience of interacting with musical content, despite levels of frustration witnessed in many interaction accounts. Repeated instances of confusion witnessed after exploratory interaction pose many design implications for consideration in novice and expert systems for interactive music in VR. Addressing these issues could allow for creating spaces with high conditions for flow, with expressive experiences free from situated distractions.

Future research directions based on findings that would benefit the domain of VR interactive music systems include: (i) Scalable frameworks for interaction analysis, such as an automatic logging system utilising human-in-the-loop machine learning; (ii) Spatial interaction related to music interfaces; (iii) Development of understanding in problem areas for music interfaces that effect flow, potentially linking flow and presence conceptualisations using physiological data; (iv) Development of questionnaires to quickly assess VR interactive music systems prototypes and artefacts.

Acknowledgements. We would like to give thanks to Stuart Cupit and the development team at Inition for guiding the initial technical development of the interface. This project was funded by the EPSRC and AHRC Centre for Doctoral Training in Media and Arts Technology (EP/L01632X/1), and the EU H2020 research and innovation project Audio Commons (688382).

\section{References}

1. Adams, A.T., Gonzalez, B., Latulipe, C.: SonicExplorer: Fluid exploration of audio parameters. In: Proc. CHI. pp. 237-246 (2014)

2. Andersen, K., Gibson, D.: The Instrument as the Source of new in new Music. In: Research Through Design. pp. 25-27 (2015) 
3. Andolina, S., Klouche, K., Cabral, D., Ruotsalo, T., Jacucci, G.: InspirationWall: Supporting Idea Generation Through Automatic Information Exploration. In: Proc. C\&C. pp. 8-11 (2015)

4. Barthet, M., Thalmann, F., Fazekas, G., Sandler, M., Wiggins, G.: Crossroads : Interactive Music Systems Transforming Performance, Production and Listening. In: Proc. CHI Workshop on Music and HCI (2016)

5. Bengler, B., Bryan-Kinns, N.: Designing collaborative musical experiences for broad audiences. In: Proc. C\&C. p. 234. ACM Press, New York, New York, USA (2013)

6. Berthaut, F., Desainte-Catherine, M., Hachet, M.: DRILE: an immersive environment for hierarchical live-looping. In: Proc. NIME. pp. 192-197 (2010)

7. Blaine, T., Fels, S.: Collaborative Musical Experiences for Novices. Journal of New Music Research (Aug 2010)

8. Bowman, D.a., Johnson, D.B., Hodges, L.F.: Testbed Evaluation of Virtual Environment Interaction Techniques. Presence: Teleoperators and Virtual Environments 10(1), 75-95 (2001)

9. Braun, V., Clarke, V.: Using thematic analysis in psychology. Qualitative research in psychology 3(2), 77-101 (2006)

10. Byrne, D.: How music works. Canongate Books (2012)

11. Cherry, E., Latulipe, C.: Quantifying the Creativity Support of Digital Tools through the Creativity Support Index. ACM ToCHI 21(4), 1-25 (Aug 2014)

12. Csikszentmihalyi, M.: Flow: the psychology of optimal experience. Harper \& Row, New York, 1 edn. (1990)

13. Elblaus, L., Hansen, K.F., Unander-Scharin, C.: Artistically Directed Prototyping in Development and in Practice. Journal of New Music Research 41(4, SI), 377-387 (2012)

14. Forlizzi, J., Battarbee, K.: Understanding Experience in Interactive Systems. In: Proc. DIS. pp. 261-268 (2004)

15. Gelineck, S.: Virtual Reality Instruments capable of changing Dimensions in Realtime. In: Proc. ENACTIVE (2005)

16. Godøy, R.I.: Gestural-Sonorous Objects: embodied extensions of Schaeffer's conceptual apparatus. Organised Sound 11(02), 149 (2006)

17. Hamilton, R.: Mediated Musical Interactions in Virtual Environments. In: CHI Workshop on Music and HCI (2016)

18. Hartmann, T., Wirth, W., Schramm, H., Klimmt, C., Vorderer, P., Gysbers, A., Böcking, S., Ravaja, N., Laarni, J., Saari, T., et al.: The spatial presence experience scale. Journal of Media Psychology (2015)

19. Heath, C.C., Luff, P.: Collaboration and control: Crisis Management and Multimedia Technology. CSCW 1 (1-2)(1990), 69-94 (1992)

20. Houde, S., Hill, C.: What do prototypes prototype. Handbook of human-computer interaction 2, 367-381 (1997)

21. Jacob, M., Magerko, B.: Interaction-based Authoring for Scalable Co-creative Agents. In: Proc. of the ICCC. pp. 236-243 (2015)

22. Johnston, A.: Beyond Evaluation : Linking Practice and Theory in New Musical Interface Design. In: Proc. NIME (2011)

23. Jordan, B., Henderson, A.: Interaction Analysis: foundations and practice. Journal of the Learning Sciences 4(1), 39-103 (1995)

24. Mulder, A., Fels, S.S., Mase, K.: Mapping virtual object manipulation to sound variation. IPSJ Sig Notes 97(122), 63-68 (1997)

25. O'hara, K., Harper, R., Mentis, H., Sellen, A., Taylor, A.: On the naturalness of touchless: putting the interaction back into NUI. ACM ToCHI 20(1), 1-25 (2013) 
26. Rodet, X., Lambert, J.p., Gaudy, T., Gosselin, F.: Study of haptic and visual interaction for sound and music control in the Phase project. In: Proc. NIME. pp. 109-114 (2005)

27. Ryan, W., Street, E., Siegel, M.a.: Evaluating Interactive Entertainment using Breakdown : Understanding Embodied Learning in Video Games. In: Proc. DiGRA (2009)

28. Salisbury, J.H., Tomlinson, P.: Reconciling Csikszentmihalyi's Broader Flow Theory: With Meaning and Value in Digital Games. ToDiGRA 2(2), 55-77 (2016)

29. Schubert, T.W.: A new conception of spatial presence: Once again, with feeling. Communication Theory 19, 161-187 (2009)

30. Sèdes, A., Guillot, P., Paris, E., Anne, S., Pierre, G., Eliott, P.: The HOA library, review and prospects. In: Proc. ICMC. vol. 2014, pp. 855-860 (2014)

31. Seldess, Z.: MIAP: Manifold-Interface Amplitude Panning in Max/MSP and Pure Data. In: Proc. AES pp. 1-10 (2011)

32. Slater, M., Usoh, M.: Body centred interaction in immersive virtual environments. Artificial life and virtual reality 1, 1-22 (1994)

33. Slepian, M.L., Ambady, N.: Fluid movement and creativity. Journal of Experimental Psychology 141(4), 625-629 (2012)

34. Stowell, D., Robertson, A., Bryan-Kinns, N., Plumbley, M.: Evaluation of live humancomputer music-making: Quantitative and qualitative approaches. International Journal of Human-Computer Studies 67(11), 960-975 (2009)

35. Sutcliffe, A., Gault, B.: Heuristic evaluation of virtual reality applications. Interacting with Computers 16(4), 831-849 (aug 2004)

36. Vinayak, Ramanujan, D., Piya, C., Ramani, K.: MobiSweep: Exploring Spatial Design Ideation Using a Smartphone as a Hand-held Reference Plane. In: Proc. TEI. pp. 12-20 (2016)

37. Wirth, W., Hartmann, T., Böcking, S., Vorderer, P., Klimmt, C., Schramm, H., Saari, T., Laarni, J., Ravaja, N., Gouveia, F.R., Biocca, F., Sacau, A., Jäncke, L., Baumgartner, T., Jäncke, P.: A Process Model of the Formation of Spatial Presence Experiences. Media Psychology 9, 493-525 (2007)

38. Wozniewski, Mike, Settel, Z., Cooperstock, J.: A spatial interface for audio and music production. In: Proc. DAFX pp. 18-21 (2006)

39. Zimmerman, J., Stolterman, E., Forlizzi, J.: An analysis and critique of Research through Design: towards a formalization of a research approach. In: Proc. DiS. pp. 310-319 (2010) 